\title{
RECORDING OF SURGICAL PROCESSES: A STUDY COMPARING \\ SENIOR AND JUNIOR NEUROSURGEONS DURING LUMBAR DISC \\ HERNIATION SURGERY
}

\author{
Laurent Riffaud ${ }^{1,2,3,4}$, M.D. \\ Thomas Neumuth ${ }^{5}$, Ph.D. \\ Xavier Morandi ${ }^{1,2,3,4}$, M.D. \\ Christos Trantakis ${ }^{5,6}$, M.D. \\ Jürgen Meixensberger ${ }^{5,6}$, M.D., Ph.D. \\ Oliver Burgert $^{5}$, Ph.D. \\ Brivael Trelhu ${ }^{2,3,4}$, M.Sc. \\ Pierre Jannin ${ }^{2,3,4}$, Ph.D.
}

\footnotetext{
${ }^{1}$ Department of Neurosurgery, Rennes University Hospital, Rennes, France

${ }^{2}$ INSERM, U746, Faculty of Medicine, Rennes, France

${ }^{3}$ INRIA, VisAGeS Unit/Project, Rennes, France

${ }^{4}$ CNRS, UMR 6074, IRISA, University of Rennes I, Rennes, France

${ }^{5}$ Innovation Center Computer Assisted Surgery (ICCAS), University of Leipzig, Leipzig, Germany

${ }^{6}$ Department of Neurosurgery, Leipzig University Hospital, Leipzig, Germany
}

\section{Disclosure}

The authors have no personal financial or institutional interest in any of the materials or devices described in this article. They did not receive any funding for this work from the following organizations: National Institutes of Health, Wellcome Trust, Howard Hughes Medical Institute, or other foundations requiring open access. 
RIFFAUD ET AL. In Journal of Neurosurgery 2010

\section{Corresponding author:}

Dr Laurent Riffaud, Service de Neurochirurgie, Hôpital Pontchaillou, rue Henri Le Guilloux, 35033 Rennes cédex 9, France. E-mail: laurent.riffaud@chu-rennes.fr, tel: +332992890 26, fax: +33299284180. 


\begin{abstract}
OBJECTIVE: Evaluating surgical practice in the operating room is difficult, and its assessment is largely subjective. Recording of standardized spine surgery processes was carried out to ascertain whether any significant differences in surgical practice can be observed between senior and junior neurosurgeons.
\end{abstract}

METHODS: Twenty-four procedures of lumbar discectomies were consecutively recorded by a senior neurosurgeon. In 12 cases, surgery was entirely performed by a senior neurosurgeon with the aid of a resident, and in the 12 remaining cases, surgery was performed by a resident with the aid of a senior. The data recorded were general parameters (operating time for the whole procedure and for each step), and general and specific parameters of the surgeon's activities (number of manual gestures, number and duration of actions performed, of use of the instruments, of interventions on anatomical structures). The Mann-Whitney U-test was used for comparison between the 2 groups of neurosurgeons.

RESULTS: The operating time was statistically lower for the group of senior surgeons. The seniors statistically demonstrated greater economy in time and in gestures during the closure step, for sewing, for the use of scissors, needle-holders and forceps. The senior surgeons statistically worked for a shorter time on the skin and used fewer manual gestures on the thoracolumbalis fascia. The number of changes in microscope position was also statistically lower for this group.

CONCLUSION: There is a relationship between surgical practice, as determined by a method of objective measurement using observation software, and surgical experience: gesture economy improves with seniority.

RUNNING TITLE: Recording of Surgical Processes

KEY WORDS: Discectomy, Manual activity, Skill, Surgery, Surgical practice, Surgical process, Surgical workflow 
Evaluating surgical practice in the operating room is difficult, and its assessment is largely subjective. Most attempts at evaluation of technical proficiency have focused on techniques that standardize the assessment process outside the operating room $(11,17)$. A device that can objectively and reliably quantify surgical practice could have advantages over traditional subjective evaluation, particularly as a screening tool. A system providing unbiased and objective measurement of surgical precision in the operating room, rather than just speed, could help the understanding of surgical processes and also provide a benchmark for training (7). Decline in the standard of surgical training in Europe resulting from reduction of working hours through the European working time directive, and from financial pressures to improve productivity, have reduced opportunities to learn surgical skills in the operating theater. Therefore the use of process models involving precise descriptions of operating procedures may be an effective tool for the definition and understanding of the surgical process $(10,15)$.

Acquisition of surgical process models using observation software, the Surgical Workflow Editor, has been validated in a context of simulated procedures for endosopic sinus surgery (15). Thus, recording of surgical processes with high accuracy has been established either for video, or live observations carried out by trained medical students. This report presents data recorded using this software, and analysis of removal of lumbar disc herniation in terms of surgical experience. The specific questions addressed in this study were firstly the feasibility of using the Surgical Workflow Editor in the context of live surgery, and secondly whether any significant differences in surgical practice can be observed between senior and junior neurosurgeons, in order to establish an objective series of criteria to serve as a basis both for assessment of performance and for the creation of pedagogical tools.

\section{MATERIALS AND METHODS}


Twenty-four neurosurgical procedures of removal of lumbar disc herniation were consecutively recorded in the Neurosurgery Department of the Leipzig University Hospital, Germany, between June and September 2008. The patients were 10 men and 14 women with a median age of 52 years (range, 21 to 72 years). These were exclusively patients with newly diagnosed disc herniation, no patient had undergone previous lumbar spine surgery which might be supposed to increase surgical difficulties due to fibrosis. The disc herniations were located as follows: L5S1 level: 13 cases (right/left = 8/5), L4L5 level: 8 cases (right/left = 6/2), L3L4 level: 2 cases (right/left = 1/1), L2L3 right: 1 case. In 12 cases, surgery was entirely performed by a senior neurosurgeon with the aid of a resident, and in the 12 remaining cases, surgery was performed by a junior neurosurgeon (resident) with the aid of a senior neurosurgeon. In these latter cases and when the junior surgeon experienced difficulties, the senior surgeon contributed to the surgical procedure by performing a part of the procedure.

\section{Description of the actors}

Surgery was performed by 5 different senior neurosurgeons and 5 different junior neurosurgeons. The senior neurosurgeons were considered as experts, each of them having performed more than 100 removals of lumbar disc herniation. The juniors were neurosurgical residents who had performed more than 2 years of their residency program. The two younger residents were in their third year of training and the three older residents in their seventh year. All the surgeons were right-handed.

\section{Description of the operative procedure}

The herniated disc was approached via a posterior intermyolamar route. The patient was installed in the genu pectoral position for this surgical procedure. Fluoroscopy was first carried out to define the precise level for the skin incision. The skin incision was made 
midline and the thoracolumbalis fascia incised on the side of the herniated disc. Muscles were then moved away from the spinous processes to allow the placing of an autostatic retractor. Another lateral x-ray image was taken before removal of the ligamentum flavum to confirm the level. This removal was performed under microscope (Zeiss Pentero microscope, Carl Zeiss, Germany) as was the dissection and removal of the herniated disc, and the hemostasis. Closure was achieved without a microscope by sewing first the thoracolumbalis fascia and then the skin.

\section{Description of acquisition of the surgical processes}

The data were acquired using a JAVA software application, the Surgical Workflow Editor $(13,14)$. A screenshot of the Surgical Workflow Editor is shown in Figure 1. The mode of data acquisition was a live observation and not a video, and the observer was a senior neurosurgeon (LR). Recordings of the surgical processes for the present study started after training of the observer (LR) in the use of the Surgical Workflow Editor. This training session concerned both lumbar discectomies and other neurosurgical procedures performed in the Neurosurgery Departments of the University Hospitals of Leipzig and Rennes. The observer (LR) who recorded the surgical process was present in the operating room and performed the recordings while the surgeon was operating. A touch-screen laptop was used to facilitate the recording task.

The data structure contained a data body subdivided into tasks representing the surgical gestures. Each task had the following structure: (1) a time element containing the subelements: start, stop and duration, (2) an actor: the surgeon, and elements indicating the various body parts and functions used: vision, right hand, left hand, (3) the activity carried out by the surgeon, (4) the instruments used, (5) the anatomical structure treated. The activities represented the manual and visual work steps performed by the neurosurgeon during the surgical procedure. The specific content ontology of the surgical process observed (surgical 
actions, instruments and anatomical structures) was adapted to the procedure of lumbar disc herniation removal by the observer (LR), and rendered possible the recording of all the gestures involved in the entire process whatever the difficulties encountered by the surgeon.

\section{Description of the recorded data}

Using the Surgical Workflow Editor, we recorded general parameters of the surgical process, and general and specific parameters of the surgeon's activities. The general parameters of the surgical process were exclusively concerned with duration either of the entire process or of the different steps chosen, whereas the general and specific parameters of the surgeon's activity concerned both the cumulative duration of time and the number of activities. The recording of duration of the entire process or of the different steps therefore took into account both the time occupied by the manual activities of the surgeon, and the intervals of time between activities.

- General parameters of the procedure: The operating time was recorded from the starting

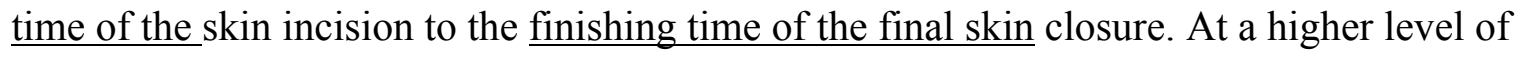
detail, the procedure of lumbar disc herniation removal was divided and recorded into 4 steps: (1) time of approach to the spine (from skin incision to the incision of the posterior longitudinal ligament or the removal of an excluded portion of the disc, (2) time of disc removal (from the end of the previous step to the beginning of hemostasis or closure), (3) time of hemostasis (this step may be not individualized if it was not performed), and (4) closure time (from the end of disc removal or hemostasis to the end incision closure).

- General parameters of the surgeon's activity: for the whole procedure and for each of the 4 different steps, the number of activities performed with either the right or the left hand, and the length of time each hand worked (total time of manual activities), were recorded. The operating time using the operating microscope and the number of times the surgeon 
changed the microscope position were recorded separately, as a visual activity, as well as the duration of fluoroscopy use after the skin incision.

- Specific parameters of the surgeon's activity: all the gestures performed by either the right or the left hand of the surgeon were recorded separately by means of a verb: coagulate, cut, dissect, drill, hold, install, irrigate, remove, sew, swab. The instruments used to perform these activities were subdivided into generic groups to avoid too much data. These instruments were: scalpels, scissors, dissectors, rongeurs (Kerrison punches), hooks, high-speed drills, suction tubes, needle-holders, saline solution, cottonoids, bone wax. Forceps were either bipolar, cup or tissue forceps. The anatomical structures treated were subdivided as follows: skin, fascia, muscle, ligament, disc, nerve root, dura mater, vertebra. No distinction was made between the different types of ligaments (the ligamentum flavum and the posterior longitudinal ligament). For the use of the operating microscope, the activity was recorded as follows: install/microscope/anatomical structure. For fluoroscopy, the activity was: install/fluoroscopy/vertebra.

\section{Analysis of data}

Results showed a nonparametric distribution. The Mann-Whitney U-test was used for comparison between the groups of senior and junior neurosurgeons. A $P$ value of less than 0.05 was deemed significant.

\section{RESULTS}

The time taken and the number of hand movements required to perform the lumbar discectomies are respectively shown in Tables 1 and 2. For the whole procedure and for each step, the number of activities performed either with the right or the left hand and the length of time each hand was active are also presented. The results demonstrated that the operating time was statistically lower for the group of senior surgeons $(\mathrm{p}<0.039)$. The difference between 
the 2 groups of surgeons was statistically significant both in the time taken $(p<0.006)$ and in the number of activities performed $(\mathrm{p}<0.031)$ to achieve the closure step, with the senior surgeons demonstrating greater economy in time and in gestures during this step.

Furthermore, the right hand worked a shorter time $(\mathrm{p}<0.039)$ and the left hand carried out fewer activities $(\mathrm{p}<0.016)$ in the group of senior surgeons.

The time taken and the number of actions (recorded by using a verb) performed either with one or the other hand during the whole procedure are presented in Table 3 . The results showed statistically significant differences for sewing, with the senior surgeons demonstrating greater economy in time $(\mathrm{p}<0.024)$ and in number of gestures $(\mathrm{p}<0.014)$ to complete this action.

The time taken and the number of times the instruments were used during the entire procedure are presented in Table 4 . The results showed statistically significant differences for the use of scissors and needle-holders, with the senior surgeons demonstrating greater economy both in time and in number of gestures. The time of use for forceps (all types of forceps considered) was statistically shorter in the group of senior surgeons $(p<0.005)$, with a shorter time of use for tissue forceps, although non significant $(p=0.078)$.

Results for microscope use are summarized in Table 5. The results showed that the number of changes in microscope position was statistically lower for the group of the senior surgeons $(\mathrm{p}<0.019)$, although the time of its use was not statistically different between the 2 groups.

The times and number of gestures concerning intervention on the anatomical structures during the whole procedure are presented in Table 6 . The results showed that the senior surgeons statistically worked a shorter time on the skin $(\mathrm{p}<0.001)$ and used fewer manual gestures on the thoracolumbalis fascia $(\mathrm{p}<0.006)$.

\section{DISCUSSION}


This study first aimed to gauge the feasibility of using Surgical Workflow Editor to produce an accurate recording of the manual gestures of neurosurgeons during a standardized surgical procedure and to carry out a specific analysis of this recording according to surgical experience.

Results showed that the mean time necessary for the entire surgical procedure was statistically shorter for the senior surgeon group. The detailed analysis demonstrated that the duration of the first three steps in the procedure (approach, discectomy, hemostasis) was shorter for the senior surgeon group without being statistically significant, and that only the final closure step was significantly shorter in this group. These results may be explained by the participation of the senior surgeon in the surgical procedures carried out by the junior: helping him when in difficulty and thus carrying out part of the procedure for him. This probably reduced the duration of the three steps concerned (approach, discectomy, hemostasis). The closure step was the most representative of the manual activity of the surgeon concerned, since it was entirely performed by the senior surgeon in the senior group and by the junior in the junior group. During this step it was therefore possible to record the manual activity of the surgeons in identical conditions, measure them and compare them in the context of an identical surgical gesture. The differences observed between senior and junior surgeons concerned both the duration of the step and the number of gestures performed. The closure step was observed to be significantly shorter in the senior surgeon group, particularly for those activities performed by the right hand, and the total number of gestures was significantly lower for the senior group. It was also possible to establish in detail that the saving in time and in number of gestures observed among the senior group specifically concerned: sewing, the use of scissors, needle-holders and forceps, and the skin and lumbosacral fascia. These results seem coherent, since the differences concerned not only the principal activity carried out during the closure step, but also the instruments and anatomical structures involved in this step. Another interesting finding involved the use of the 
microscope, the number of position changes being twice as great in the junior group, although the overall time of use did not vary significantly from one group to the other. This observation confirms the fact that the differences recorded between the two groups during the surgical procedure involved not only the length of time taken by the different activities but also the number of gestures. A clear tendency was observed among the senior surgeons to use fewer gestures than the juniors to carry out the same task. The results for microscope use are a good illustration of the economy of gestures observed among senior surgeons, and of the less welldeveloped technique of juniors, whose over-frequent adjustments of microscope position have a negative effect on the manual gestures being carried out: interruption of the surgeon's concentration and the fluidity of the gesture. The consequence is an obstacle to the optimisation of the activity.

The question remains whether the results observed really reflect a difference in manual dexterity between the two groups of surgeons with different levels of experience. Manual dexterity of surgeons has been mostly analysed away from the operating theater using laboratory simulation in both open and laparoscopic models as well as in virtual reality (11, $12,17)$. There were several reasons for using simulations rather than evaluations within the operating theater to assess skill (9). For example, inanimate simulation with synthetic models allowed exact duplication of conditions for participants and more valid comparison of performance between individuals. Methods of assessing operative skill of surgeons and their trainees were both qualitative (direct observation of the task and then scoring it) $(16,18)$ and quantitative. Quantitative measurement of manual dexterity by motion analysis (time taken and number of movements) has been studied using bench test models of surgery like suturing and knot-tying tasks $(4,5,8)$. These experiments showed that more experienced surgeons performed these tasks in a shorter time than junior trainees. This has raised the question why experienced surgeons were taking less time to achieve the task. Were they performing rapid movements to complete the tasks or were they making fewer movements to generate the faster 
time? This question emphasized the fact that it was necessary to measure both the number of movements and the time to give a true reflection of dexterity. The experiments using simple bench tasks confirmed that experienced surgeons were more economical with their movements, performing the exercises with fewer moves rather than with higher speed (4). But performance within the operating theatre does not depend only on technical skill. The operating room can be a threatening and stressful environment so that surgeons may behave differently, and the transfer of observations of technical skills from laboratory to live surgery remains unanswered (1). The results from this initial study are promising and may give the beginning of a response: analysis of the manual activities of the surgeon in real surgical practice showed similar results to analysis of surgical skill in synthetic models. The experienced surgeons (senior) were more economical with their movements than the inexperienced ones (junior) during a mechanical step (closure). This observation was not so clear for activities performed during non-mechanical steps like the approach and the discectomy where the manual gestures were not different from one group to the other.

Although the operating room should be the ideal location for the assessment of surgical practice and the acquisition of primary technical skills, several reasons are currently making it difficult in our teaching institutions (3): the financial pressure that forces surgeons to be more and more efficient in the operating room, the increasing number of patients with complex surgical problems that demand the skill of expert surgeons working at maximum efficiency, and finally ethical concerns about teaching basic skills using a patient. Furthermore, the use of Surgical Workflow Editor for recording surgical processes in the operating room has some limitations. First, the need to record standardized operations for a valid comparison may involve some difficulties, as all patients are different. In this study, we recorded a frequent and reproducible surgical process and avoided recording more complicated surgical processes, such as patients with previous lumbar spine surgery for whom the procedure would be longer due to the dissection of fibrosis. This choice allowed a more accurate comparison of the 
activities between the 2 groups of surgeons and results were less difficult to interpret.

Secondly, recording surgical processes may be time-consuming in the long term. In a previous work, Neumuth et al. (15) demonstrated that trained medical students can be highly accurate observers in recording such surgical processes, which may make it possible to increase the number of routine observers for recording. Nevertheless, the Surgical Workflow Editor appeared to be a method of assessment of surgical practice appropriate for use in the context of live surgery. According to Moorthy et al. (11), a method that makes the assessment process objective should also be valid and reliable. Indeed, the assessment of technical skills by direct observation, as currently occurs in the operating room, is influenced by subjectivity: the assessment is global and not based on specific criteria. Furthermore, morbidity and mortality data, often used as surrogate markers of operative performance, are influenced by patient characteristics and do not truly reflect surgical performance (6). The availability of several criteria and parameters that can be recorded using the Surgical Workflow Editor and the opportunity to adapt them to each surgical process confirm our method of assessing surgical skills as an objective one, and complete the arsenal of methods of measurement of surgical practice and dexterity.

\section{CONCLUSION}

We have demonstrated a relationship between surgical practice, as determined by a method of objective measurement using observation software, and surgical experience in a context of live surgery: gesture economy improves with seniority. This result is encouraging and will hopefully spur further investigations into other surgical procedures, or fields of medicine that require proficiency in manual dexterity. 


\section{REFERENCES}

1. Aggarwal R, Darzi A. Technical-skills training in the $21^{\text {st }}$ century. $\mathbf{N}$ Engl J Med $355: 2695-2696,2006$

2. Aggarwal R, Grantcharov TP, Darzi A: Framework for systematic training and assessment of technical skills. J Am Coll Surg 204:697-705, 2007

3. Anastakis DJ, Regehr G, Reznick RK, Cusimano M, Murnaghan J, Bown M, Hutchison C: Assessment of technical skills transfer from the bench training model to the human model. Am J Surg 177:167-170, 1999

4. Bann S, Kahn MS, Darzi A: Measurement of surgical dexterity using motion nalysis of simple bench tasks. World J Surg 27:390-394, 2003

5. Bann S, Khan MS, Datta VK, Darzi AW: Technical Performance: relation between surgical dexterity and technical knowledge. World J Surg 28:142-147, 2004

6. Bridgewater B, Grayson AD, Jackson M, Brooks N, Grotte GJ, Keenan DJM, Millner R, Fabri BM, Jones M: Surgeon specific mortality in adult cardiac surgery: comparison between crude and risk stratified data. BMJ 327:13-17, 2003

7. Darzi A, Smith S, Taffinder N: Assessing operative skill. Needs to become more objective. BMJ 318:887-888, 1999

8. Datta V, Mandalia M, Mackay S, Chang A, Cheshire N, Darzi A: Relationship between skill and outcome in the laboratory-based model. Surgery 131:318-323, 2002

9. Datta V, Bann S, Beard J, Mandalia M, Darzi A: Comparison of bench test evaluations of surgical skill with live operating performance assessments. J Am Coll Surg 199:603606,2004

10. Jannin P, Morandi X: Surgical models for computer-assisted neurosurgery. Neuroimage 37:783-791, 2007 
11. Moorthy K, Munz Y, Sarker SK, Darzi A: Objective assessment of technical skills in surgery. BMJ 327:1032-1037, 2003

12. Neequaye SK, Aggarwal R, Van Herzeele I, Darzi A, Cheshire NJ: Endovascular skills training and assessment. J Vasc Surg 46:1055-1064, 2007

13. Neumuth T, Durstewitz N, Fischer M, Strauss G, Dietz A, Meixensberger J, Jannin P, Cleary K, Lemke HU, Burgert O: Structured recording of intraoperative surgical workflows, in Horii SC, Ratib OM (eds): SPIE Medical Imaging 2006: PACS and Imaging Informatics. Bellingham, 2006, p CID 61450A

14. Neumuth T, Mudunuri R, Jannin P, Meixensberger J, Burgert O: SWAN-Suite: The tool landscape for surgical workflow analysis, in Troccaz J, Merloz P (eds): Computer Assisted Medical and Surgical Interventions (SURGETICA). Paris, Sauramps Medical, 2007, pp 199-204

15. Neumuth T, Jannin P, Strauss G, Meixensberger J, Burgert O: Validation of knowledge acquisition for surgical process models. J Am Med Inform Assoc 16:72-80, 2009

16. Reznick R, Regehr G, MacRae H, Martin J, McCulloch W: Testing technical skill via an innovative "bench station” examination. Am J Surg 173:226-230, 1997

17. Reznick RK, MacRae H: Teaching surgical skills-changes in the wind. N Engl J Med $355: 2664-2669,2006$

18. Winckel CP, Reznick RK, Cohen R: Reliability and construct validity of a structured technical skills assessment form. Am J Surg 167:423-427, 1994

\section{ACKNOWLEDGMENTS}

The authors thank Mrs Deirdre McKeown for her help with the English language 
RIFFAUD ET AL. In Journal of Neurosurgery 2010

\section{FIGURE LEGENDS}

FIGURE 1. Screenshot of the Surgical Workflow Editor. 